\title{
Diacronie
}

Studi di Storia Contemporanea

$\mathrm{N}^{\circ} 26,2$ | 2016

Un bilancio della scommessa democratica della Chiesa cattolica

\section{Luca G. Manenti, Da Costantinopoli a Trieste, vita di Gregorio Ananian, medico e benefattore armeno}

\section{Luca Zuccolo}

\section{(2) OpenEdition \\ Journals}

Edizione digitale

URL: http://journals.openedition.org/diacronie/4038

DOI: $10.4000 /$ diacronie.4038

ISSN: 2038-0925

\section{Editore}

Association culturelle Diacronie

Notizia bibliografica digitale

Luca Zuccolo, « Luca G. Manenti, Da Costantinopoli a Trieste, vita di Gregorio Ananian, medico e

benefattore armeno », Diacronie [Online], № 26, 2 | 2016, documento 14, Messo online il 29 juin 2016, consultato il 24 septembre 2020. URL : http://journals.openedition.org/diacronie/4038 ; DOI : https:// doi.org/10.4000/diacronie.4038 
N. 26 | 2|2016 Un bilancio della scommessa democratica della Chiesa cattolica

14/

\section{RECENSIONE:}

\section{Luca G. MANENTI, Da Costantinopoli a} Trieste, vita di Gregorio Ananian, medico e benefattore armeno, Milano, Biblion edizioni, 2015, 122 pp.

a cura di Luca ZUCCOLO *

La vita di ogni uomo è ricca di sfaccettature, di scelte e cambiamenti, spesso radicali che la trasformano, che la rendono diversa per sé e per gli altri, al pari dei popoli, dei gruppi sociali o delle congregazioni religiose. Questa molteplicità di piani, a volte impervi, rappresenta la vita di ciascuno di noi ed è anche il centro della storia di Gregorio Ananian (1770-1865), raccontata nel recente saggio di Luca Manenti, storico di formazione milanese, ma attualmente borsista presso l'università di Trieste' ${ }^{1}$.

Il volume, patrocinato dalla Fondazione Ananian di Trieste in concomitanza con il $150^{\circ}$ anniversario della morte del suo fondatore, non ha solo uno scopo elogiativo e celebrativo, bensì l'intento di «costruire [...] parti del futuro di tutti noi», come ricorda il presidente della fondazione Giovanni Damiani. La celebrazione, infatti, permette di «parlare della volontà di tessere un racconto universale dello straordinario potere della conoscenza, di come lo studio e il sapere siano il motore che permette l'avanzata sociale del singolo e della collettività». Una collettività che è quella armena, quella ottomana, asburgica e, infine, quella triestina che per le sue peculiarità rende la città giuliana «una città “esperimento della storia" ${ }^{2}$.

\footnotetext{
${ }^{1}$ L'autore si occupa principalmente di associazionismo, massoneria e minoranze religiose tra XVIII e XX secolo, con particolare attenzione alla regione dell'alto-Adriatico. Tra i suoi saggi più recenti si annovera il volume Massoneria e Irredentismo. Geografia dell'associazionismo patriottico in Italia tra Otto e Novecento, Istituto regionale per la storia del movimento di liberazione nel Friuli Venezia Giulia, Trieste, 2015.

2 DAMIANI, Giovanni, Prefazione, in MANENTI, Luca G., Da Costantinopoli a Trieste, cit., pp. 7-10.
} 
Se Trieste è il punto d'arrivo della vita del filantropo Ananian, ben più lontane sono le sue radici armene, da cui l'autore inizia il suo racconto, legando indissolubilmente il protagonista del saggio alla storia di questo popolo. L'agile e ricco volume di Manenti, infatti, ha come fine quello «di restituire questo intricato scenario, di cui la biografia di Ananian ha rappresentato il filo rosso, in grado di congiungere tutti i nodi tematici evocati»3. Pertanto, il racconto della vita di Gregorio inizia proprio dalla storia armena tratteggiata con maestria nei suoi caratteri principali e nelle sue evoluzioni ottocentesche. Una storia che dalla Cilicia attraverso l'Impero Ottomano, Venezia e Vienna giunge sino a Trieste, doppiando, in una chiave più estesa, quanto personalmente compie il medico ottomano Ananian, il quale, dopo aver studiato a Parigi e Padova, dall'Istanbul di Selim III - dove esercitava la pratica medica appresa in Europa in qualità di ginecologo dell'Harem imperiale - si trasferisce nella città giuliana.

Di notevole interesse in questo racconto che non è solo una biografia o una storia del popolo armeno descritto attraverso un suo esimio rappresentante, è anche storia sociale e culturale. La vita e le opere di Gregorio testimoniano chiaramente quali fossero le temperie culturali e sociali dell'epoca e come il dialogo tra modernità e tradizione non fosse sempre lineare e chiaro.

Ananian, infatti, si trovò completamente immerso in questo ambiente culturale durante gli anni di attività come ginecologo imperiale a Istanbul. In questo periodo accanto alla scienza medica e alle sue evoluzioni ed innovazioni testimoniate dagli stessi studi sulle sostanze oppiacee sviluppati da Ananian, il medico armeno esperì direttamente il grande mutamento sociale e politico voluto dal Sultano Selim III, il quale con il suo "ordine nuovo" (Nizam-i cedid) iniziò un lungo periodo di riforme che stravolsero l'Impero Ottomano, costrinsero Ananian alla precipitosa fuga, ma lo influenzarono ampiamente dal punto di vista umano e sociale. Influenza poi trasmessa nella sua attività filantropica.

Altrettanto importante in questo continuo passaggio tra il piano biografico del singolo e quello storico del gruppo, è il ruolo giocato dai monaci mechitaristi prima a Venezia poi a Trieste e delle colonie armene in Italia, appunto, e nell'Impero asburgico. Questi due fattori, determinanti per la stabilizzazione di Ananian a Trieste definiscono su più ampia scala quale fu il ruolo delle comunità armene nelle terre veneziane $\mathrm{e}$ giuliane. Un ruolo strettamente legato alla cultura e alla sua diffusione sia per mezzo della stampa e della tipografia, sia per mezzo delle scuole. Due capisaldi anche della futura attività filantropica di Ananian, il quale dopo il trasferimento nel porto franco

3 Ibidem, p. 11. 
giuliano, entra subito a far parte non solo della comunità armena ivi presente, ma anche della società e della sociabilità urbana triestina.

Transitando dal piano ampio e transnazionale degli imperi e della comunità Armena, infatti, l'autore ci trasporta in quella che sarà l'ultima realtà di vita e professionale di Ananian, Trieste, città portuale e punto d'arrivo e partenza non solo di mezzi e uomini, ma anche di cultura, religione e idee. Città meticcia e multiculturale in cui il "benefattore venuto d'Oriente" sin da subito ricoprirà incarichi di rilievo divenendo anche consigliere comunale4.

Nondimeno, l'attività filantropica sarà la pietra miliare della vita e del ricordo di Gregorio Ananian, il quale tramite la sua fondazione, a cui per legato testamentario donò tutte le sue fortune, diede ampio spazio alla beneficienza verso i poveri e allo sviluppo della cultura. Attività filantropica che lo renderà un beniamino della popolazione triestina e non solo, come dimostra l'ampio cordoglio dei suoi concittadini alla sua morte e il gran numero di altre fondazioni che negli anni si sono unite o sono state trasferite nella loro gestione sotto l'egida della fondazione Ananian. L'attenzione per i meno fortunati, infatti, rappresentò nel XIX secolo una sorta di «calmierante sociale»5 sviluppato dalle classi abbienti per dimostrare il loro altruismo e per redistribuire le ricchezze all'interno del tessuto urbano. Un altruismo che si perpetuerà anche nel secolo successivo proprio grazie alla fondazione di Gregorio, che continuerà a diffondere con le opere e con i fatti il messaggio del suo fondatore.

Il lavoro di Manenti è tutto questo e molto altro ancora. Il volume, infatti, si completa con un apparato metatestuale molto accurato in cui spiccano la bibliografia delle fonti primarie e secondarie e gli indici analitici. Lustro e merito ancor maggiore di questo libro sono, infine, i documenti riprodotti dall'autore in appendice. Una serie di documenti originali che accrescono la comprensione della vita di un esimio rappresentante del popolo Armeno, della città di Trieste e dell'umanità tutta. Di certo interesse, sono il documento numero 3 che descrive le opinioni di Gregorio Ananian sull'oppio e sulle sue funzioni curative, testimonianza di studi medici "innovativi", seppur oggi poco percorribili ${ }^{6}$, e il documento numero 9 , in cui viene riportato per intero il suo testamento, testimonianza ed esplicitazione della sua attività filantropica e umanitaria7, nonché lascito tangibile delle sue ultime volontà a favore dei meno abbienti.

\footnotetext{
4 Arrivato a Trieste dopo una fuga precipitosa da Istanbul ed inseritosi nel tessuto sociale del porto giuliano, Gregorio Ananian si dedicò dal 1843 al 1848 anche alla vita politica cittadina.

5 Ibidem, p. 44.

${ }^{6}$ Ibidem, pp. 64-68.

7 Ibidem, pp. 83-90.
} 
Sin dalle prime pagine di questo ottimo saggio, il fine commemorativo si muta in un affresco della vita di Gregorio Ananian e del popolo armeno tutto, ricordando e mettendo in evidenza l'importante ruolo culturale, sociale e intellettuale svolto dai rappresentanti di questa comunità e in modo peculiare dai gruppi della diaspora armena. Figure come quella di Ananian hanno dimostrato, pur nella loro unicità, uno spessore che va ben al di là della semplice filantropia per innalzarsi - come si dice nell'introduzione e nelle pagine del saggio di Manenti - ad epitome dello sviluppo socio-culturale dell'intera comunità. Per queste ragioni e per la pregevole scrittura di Luca Manenti il suo breve saggio su Gregorio Ananian diventa una lettura interessante ed imprescindibile per chiunque voglia approcciarsi alla figura di Ananian, ai protagonisti della storia del popolo Armeno e anche alla storia urbana e del tessuto socio-culturale della città di Trieste. 


\section{* L'autore}

Luca Zuccolo, dottore (PhD) in Storia Contemporanea del SUM di Napoli ha sviluppato una ricerca sulle dinamiche di formazione dell'identità ottomana e di un discorso di tipo patriottico durante il regno di Abdülhamid II (1880-1885) attraverso lo studio di fonti a stampa redatte in lingua francese. Già dottore magistrale in Storia d'Europa (Bologna 2008), si è occupato dello sviluppo della modernità durante l'ultimo secolo dell'Impero Ottomano, del confronto/scontro tra modernità e tradizione in un contesto cosmopolita e allo sviluppo dei movimenti sociali che hanno preparato l'avvento della società turca contemporanea.

URL: < http://www.studistorici.com/2010/12/o1/luca-zuccolo/ >

\section{Per citare questo articolo:}

ZUCCOLO, Luca, «Recensione: Luca G. MANENTI, Da Costantinopoli a Trieste, vita di Gregorio Ananian, medico e benefattore armeno, Milano, Biblion edizioni, 2015, 122 pp.», Diacronie. Studi di Storia Contemporanea : Un bilancio della scommessa democratica della Chiesa cattolica, 29/06/2016,

URL:< http://www.studistorici.com/2015/06/29/zuccolo_numero_26/ >

Diacronie Studi di Storia Contemporanea $\beta$ ww.diacronie.it

Risorsa digitale indipendente a carattere storiografico. Uscita trimestrale. redazione.diacronie@hotmail.it

Comitato di redazione: Jacopo Bassi - Luca Bufarale - Elisa Grandi - Antonio César Moreno Cantano - Deborah Paci - Fausto Pietrancosta - Alessandro Salvador - Matteo Tomasoni - Luca Zuccolo

Diritti: gli articoli di Diacronie. Studi di Storia Contemporanea sono pubblicati sotto licenza Creative Commons 3.0. Possono essere riprodotti e modificati a patto di indicare eventuali modifiche dei contenuti, di riconoscere la paternità dell'opera e di condividerla allo stesso modo. La citazione di estratti è comunque sempre autorizzata, nei limiti previsti dalla legge. 